\title{
Negative differential thermal resistance and thermal transistor
}

\author{
Baowen $\mathrm{Li}^{\mathrm{a})}$ \\ Department of Physics, National University of Singapore, Singapore 117546, Republic of Singapore; \\ Center for Computational Science and Engineering, National University of Singapore, Singapore 117542, \\ Republic of Singapore; Laboratory of Modern Acoustics, Institute of Acoustics, Nanjing University, \\ Nanjing 210093, People's Republic of China; and NUS Graduate School for Integrative Sciences \\ and Engineering, Singapore 117597, Republic of Singapore
}

Lei Wang

Department of Physics, National University of Singapore, Singapore 117546, Republic of Singapore and Center for Computational Science and Engineering, National University of Singapore, Singapore 117542, Republic of Singapore

\begin{abstract}
Giulio Casati
Department of Physics, National University of Singapore, Singapore 117546, Republic of Singapore; Center for Computational Science and Engineering, National University of Singapore, Singapore 117542, Republic of Singapore; International Center for Nonlinear and Complex Systems, Universita' degli studi dell'Insubria, 22100 Como, Italy; Istituto Nazionale di Fisica della Materia, Unita' di Como, 22100 Como, Italy; and Istituto Nazionale di Fisica Nucleare, Sezione di Milano, I-20133 Milano, Italy
\end{abstract}

(Received 9 January 2006; accepted 7 March 2006; published online 3 April 2006)

\begin{abstract}
We report on the first model of a thermal transistor to control heat flow. Like its electronic counterpart, our thermal transistor is a three-terminal device with the important feature that the current through the two terminals can be controlled by small changes in the temperature or in the current through the third terminal. This control feature allows us to switch the device between "off" (insulating) and "on" (conducting) states or to amplify a small current. The thermal transistor model is possible because of the negative differential thermal resistance. (C) 2006 American Institute of Physics. [DOI: 10.1063/1.2191730]
\end{abstract}

The invention of the transistor ${ }^{1}$ and other relevant devices that control the electric charge flow has led to an impressive technological development. In recent years some interesting progress has been made also in the control of the heat current. Indeed a theoretical model of a thermal rectifier has been recently proposed ${ }^{2}$ in which the heat can flow preferentially in one direction. More recently, new models of thermal diode have been devised ${ }^{3,4}$ which allow to improve the efficiency by more than three orders of magnitude.

In this Letter we propose a model for a thermal transistor which can control the heat flow in analogy to the usual electric transistor for the control of the electric current. The reason why such a device can, in principle, operate is grounded in the phenomenon of negative differential thermal resistance (NDTR) which, as shown below, can take place in nonlinear lattices.

Our model of thermal transistor consists of three segments, $D, S$, and $G$ [see Fig. 1(a) for its configuration]. The names $D, S$, and $G$ follow from the ones used in a metaloxide-semiconductor field-effect transistor (MOSFET). Each segment is a Frenkel-Kontorova (FK) lattice. ${ }^{5,6}$ Segments $S$ and $D$ are coupled (usually weakly) via a spring of constant $k_{\text {int }}$, while segment $G$, the control segment, is coupled to the junction particle $O$ between segments $S$ and $D$ via a spring of constant $k_{\text {int }_{G}}$. Temperature $T_{G}$ is used to control temperature $T_{O}$ (at the junction $O$ ) which determines the heat current from $D$ to $S$. The total Hamiltonian of the model writes

\footnotetext{
${ }^{a)}$ Electronic mail: phylibw@nus.edu.sg
}

$$
\begin{aligned}
H= & H_{S}+H_{D}+H_{G}+H_{\mathrm{int}}+H_{\mathrm{int}_{G}}=\sum_{i=1}^{N_{S}} \frac{1}{2} \dot{x}_{S, i}^{2}+\frac{1}{2} k_{S}\left(x_{S, i}\right. \\
& \left.-x_{S, i-1}\right)^{2}-\frac{V_{S}}{(2 \pi)^{2}} \cos 2 \pi x_{S, i}+\sum_{i=1}^{N_{D}} \frac{1}{2} \dot{x}_{D, i}^{2}+\frac{1}{2} k_{D}\left(x_{D, i}\right. \\
& \left.-x_{D, i-1}\right)^{2}-\frac{V_{D}}{(2 \pi)^{2}} \cos 2 \pi x_{D, i}+\sum_{i=1}^{N_{G}} \frac{1}{2} \dot{x}_{G, i}^{2}+\frac{1}{2} k_{G}\left(x_{G, i}\right. \\
& \left.-x_{G, i-1}\right)^{2}-\frac{V_{G}}{(2 \pi)^{2}} \cos 2 \pi x_{G, i}+\frac{1}{2} k_{\text {int }}\left(x_{S, N_{S}}-x_{D, N_{D}}\right)^{2} \\
& +\frac{1}{2} k_{\text {int }_{G}}\left(x_{S, N_{S}}-x_{G, N_{G}}\right)^{2} .
\end{aligned}
$$

Here $x_{D, i}, x_{S, i}$, and $x_{G, i}$ are the particles' displacements from their equilibrium positions in segments $D, S$, and $G$, respectively. Fixed boundary conditions are taken, i.e., $x_{D, 0}=x_{S, 0}=x_{G, 0}=0$.

In the following we will present first the results of our numerical simulations, then the theoretical explanation. In our numerical simulations the lattices are coupled, at their first particles, with heat baths at different temperatures $T_{D}$, $T_{S}$, and $T_{G}$, respectively. We use Langevin heat baths and we have checked that our results do not depend on the particular heat bath realization (e.g., Nose-Hoover heat baths). The local heat flux is defined by $J_{n} \equiv k\left\langle\dot{x}_{n}\left(x_{n}-x_{n-1}\right)\right\rangle$ and the local temperature is defined as $T_{n}=\left\langle\dot{x}_{n}^{2}\right\rangle$. The simulations are performed long enough to allow the system to reach a nonequilibrium steady state where the local heat flux is constant in each segment. 

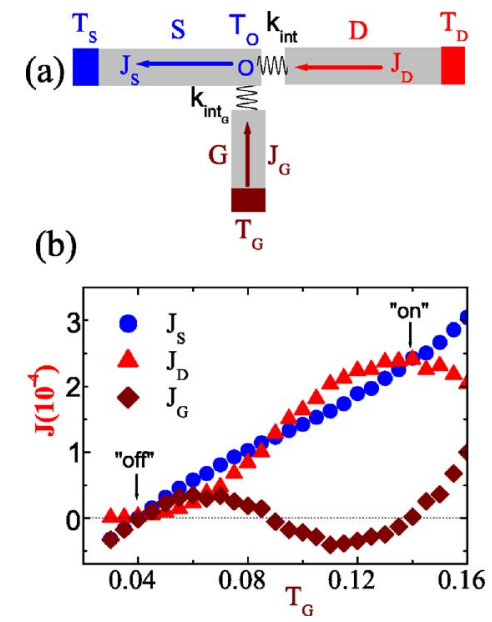

FIG. 1. (Color online) (a) Configuration of the thermal transistor. (b) Heat current vs the control temperature $T_{G}$. Parameters are $T_{D}=0.2, V_{D}=1.0$, $k_{D}=0.2, k_{\text {int }}=0.05, T_{S}=0.04, V_{S}=5, k_{S}=0.2, V_{G}=5, k_{G}=1$, and $k_{\text {int }_{G}}=1$. Notice that in a wide region both $J_{S}$ and $J_{D}$ increase when the temperature $T_{G}$ is increased.

As illustrated in Figs. 1 and 2, the model described by Hamiltonian (1) can exhibit different useful functions depending on the parameters values.

Thermal switch. We first demonstrate the switch function of our transistor, namely, we show that the system can act like a good heat conductor or an insulator depending on the control temperature $T_{G}$. This is shown in Fig. 1(b), where we plot $J_{G}, J_{S}$, and $J_{D}$ vs $T_{G}$. When $T_{G}$ increases from 0.03 to 0.135 , both $J_{D}$ and $J_{S}$ increase. In particular, at three points $T_{G} \approx 0.04,0.09$, and 0.135 , we have that $J_{D}=J_{S}$ and thus $J_{G}$ is exactly zero. These three points correspond to "off," "semi-on," and "on" states, at which $J_{D}$ is $2.4 \times 10^{-6}$, $1.2 \times 10^{-4}$, and $2.3 \times 10^{-4}$, respectively. The ratio of the heat currents at the on and off states is about 100; hence our model displays one important function-switch-just like the function of a MOSFET used in a digital circuit.

Thermal modulator/amplifier. As demonstrated above, the heat current from $D$ to $S$ can be switched between different values. However, in many cases, such as in an analog circuit, one needs to continuously adjust the current in a wide range. In Fig. 2 we demonstrate this "modulator/amplifier" function of our transistor. Here it is seen that in the temperature interval $T_{G} \in(0.05,0.135)$, the heat current through the segment $G$ remains very small $\left(-10^{-5}-10^{-5}\right)$, within the shadow strip in Fig. 2, while the heat currents $J_{S}$ and $J_{D}$ are continuously controlled from $5 \times 10^{-5}$ to $2 \times 10^{-4}$.

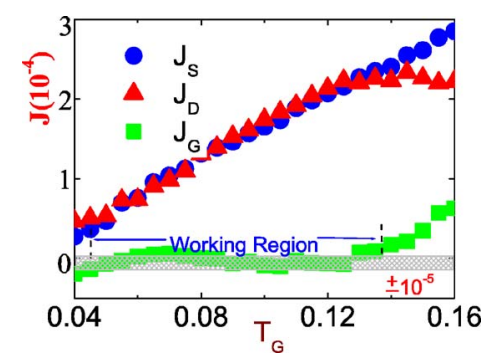

FIG. 2. (Color online) Heat current vs the control temperature $T_{G}$. Here $T_{D}=0.2, \quad V_{D}=1.0, k_{D}=0.2, T_{S}=0.04, V_{S}=5, k_{S}=0.2, \quad k_{\mathrm{int}}=0.05, \quad V_{G}=5$, $k_{G}=1$, and $k_{\text {int }_{G}}=0.1$. The shadow region is the range of variation of $J_{G}$ in

the temperature interval $T_{G} \in(0.05,0.135)$.
Downloaded 08 May 2006 to 193.206.162.158. Redistribution subject to AlP license or copyright, see http://apl.aip.org/apl/copyright.jsp
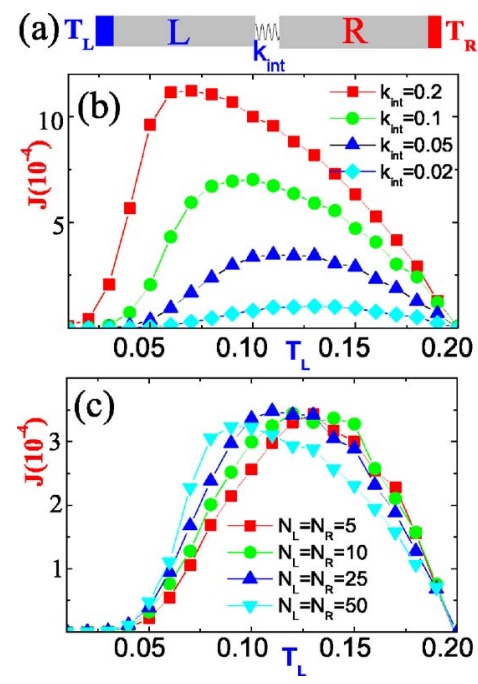

FIG. 3. (Color online) (a) Configuration of the model exhibiting the negative differential thermal resistance (see definition in the text). (b) Heat current vs temperature $T_{L}$ (at fixed $T_{R}=0.2$ ) for different coupling constants $k_{\text {int }}$, with lattice size $N_{L}=N_{R}=25$. The system parameters are $V_{L}=5, V_{R}=1$, $k_{L}=1$, and $k_{R}=0.2$. (c) Same as (b) but for different system sizes and $k_{\text {int }}=0.05$. Notice that when $T_{L} \leqslant 0.1$ the heat current increases with decreasing the external temperature difference.

Let us now turn to the discussion of the underlying physical mechanism which allows the transistor to function. In all cases considered here, the heat resistance of segment $G$ has been taken small enough so that $T_{O} \approx T_{G}$. For fixed understand how the heat currents $J_{S}$ and $J_{D}$ depend on temperature $T_{O}$. This dependence is determined by the differential thermal resistances, $R_{S}=\left(\partial J_{S} / \partial T_{O}\right)_{T_{S}=\text { const }}^{-1}$ and $R_{D}=-\left(\partial J_{D} / \partial T_{O}\right)_{T_{D}=\text { const. }}^{-1}$ In typical situations the heat current increases with the increase of the thermal gradient and therefore both $R_{S}$ and $R_{D}$ are positive. As a consequence, the current amplification factor

$$
\alpha \equiv\left|\frac{\partial J_{D}}{\partial J_{G}}\right|=\left|\frac{R_{S}}{R_{S}+R_{D}}\right|<1,
$$

namely, in order to induce a change $\Delta J_{D}$, the control heat bath has to provide a larger $\Delta J_{G}$. This means that the "transistor" can never work!

In order to get the amplification effect, namely, $\alpha>1$, either $R_{S}$ or $R_{D}$ must be negative. Thus we must have a negative differential thermal resistance (NDTR). In such case the possibility arises that, when $T_{O}$ changes both $J_{S}$ and $J_{D}$ changes simultaneously in the same way. Therefore the condition $J_{S}=J_{D}$ can be achieved for several different values of $T_{O}$ (as shown in Fig. 1). Analogously, the condition $J_{S} \approx J_{D}$ can be verified in a wide range of $T_{O}$ (as shown in Fig. 2). In these situations heat switch and heat modulator/amplifier are possible. In the ideal, limiting case of $R_{S}=-R_{D}$ which, in principle, can be obtained by adjusting parameters, the transistor works perfectly.

Figure 3 illustrates the mechanism of NDTR. In Fig. 3(b) we plot the heat current versus temperature $T_{L}$ [see Fig. 3(a)] for fixed $T_{R}$, fixed lattice size, and different coupling constants $k_{\text {int }}$. In Fig. 3(c) instead the heat current is plotted for different lattice sizes with fixed coupling constants $k_{\text {int }}$.

As it is clearly seen, in a wide range of system parameters, for a fixed temperature $T_{R}$, there exists a temperature AlP license or copyright, see http://apl.aip.org/apl/copyright.jsp thermal bath temperatures $T_{S}$ and $T_{D}$, we would like now to 

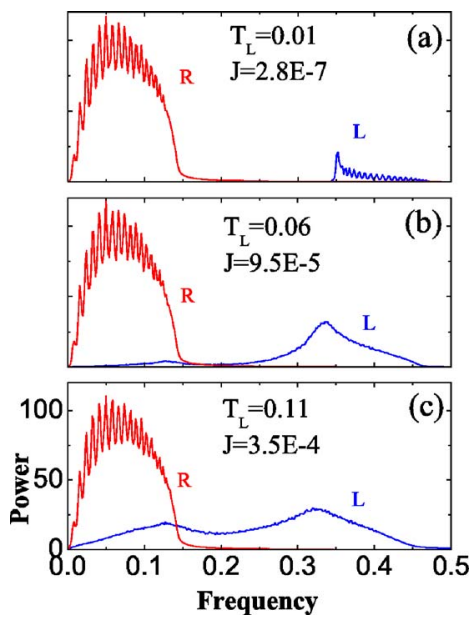

FIG. 4. (Color online) The power spectra of the particles at the right (R) side and the left $(\mathrm{L})$ side of the interface for different temperatures $T_{L}$ with fixed $T_{R}=0.2$. The system parameters are $V_{L}=5, V_{R}=1, k_{L}=1, k_{R}=0.2$, $k_{\text {int }}=0.05$, and $N=50$. Notice that the overlap of the spectra increases as the temperature $T_{L}$ increases, which results in the increase of the heat current with decreasing the external temperature difference when $T_{L} \leqslant 0.11$.

interval in which a larger value of $T_{L}\left(<T_{R}\right)$, corresponding to a smaller thermal gradient, can induce a larger heat current.

The phenomenon of NDTR can be understood from the mismatch between the phonon bands of the two interface particles. This is illustrated in Fig. 4 where we plot the power spectra of the two interface particles at different temperatures $T_{L}$ for a fixed $T_{R}=0.2[$ Fig. 3(a)]. As it is seen, the overlap of the power spectra increases as $T_{L}$ increases, i.e., as the thermal gradient decreases. Therefore, by increasing $T_{L}$ we are in the presence of two competing effects: from one hand the thermal gradient decreases and on the other hand the band overlap increases. The beavior of the heat current depends on which of these two effects prevails. In particular, the phenomenon of NDTR can take place.

The physical mechanism of such behavior is due to the fact that the variation of the band position with temperature depends on the strength of nonlinearity. This general property is at the base of the thermal diode models. ${ }^{3,4}$ In particular, for the model discussed in this Letter, as the temperature $T_{L}$ increases, the kinetic energy of the left interface particle becomes large enough to overcome the on-site potential barrier and low frequency modes appear.

Finally, we would like to discuss the possibility of building a thermal transistor in real physical systems and the possible applications. Due to the fast developing semiconductor technology, it is now possible to fabricate several low dimensional devices, such as nanotubes, nanowires, and thin films, in nanometer scale. ${ }^{7}$ A nanotube with small diameter or a nanowire is basically a one dimensional (1D) heat conductor. For the purpose of our model, which is indeed one dimensional, one can fabricate a T-shaped nanotube or nanowire.
To build the on-site potential in the FK model, one may put different segments of nanotubes/nanowires on the surface of different substrates.

Among the possible applications we would like to mention, as an example, that in our model, by adjusting the temperature of the third terminal, one can amplify the current through the other two terminals. This can be used as an efficient heat pump to dissipate heat from a microelectronic or nanoelectronic device where a large amount of redundant heat is produced due to the fast operational speed of the device. This could be very important since, as it is known, the redundant heat, if it is not dissipated immediately, may deteriorate the device.

In conclusion, we have built a theoretical model for a thermal transistor. The model displays two basic functions of a transistor: switch and modulator/amplifier. The crucial element of the thermal transistor is the negative differential thermal resistance. Although at present this is no more than an abstract model, we believe that, sooner or later, it can be realized in a nanoscale system experiment. After all the Frenkel-Kontorova model used in our simulations is a very popular model in condensed matter physics. ${ }^{5,6}$ For instance, it has been used to model crystal dislocations, epitaxial monolayers on the crystal surface, ionic conductors and glassy materials, an electron in a quasi-1D metal below the Peierls transition, charge density waves, Josephson junction chains, and dry friction. More recently, this model has been employed to study transport properties of vortices in easy flow channels ${ }^{8}$ and strain-mediated interaction of vacancy lines in a pseudomorphic adsorbate system. ${ }^{9}$

This project is supported in part by FRG of NUS and the DSTA Singapore under Project Agreement POD0410553 (B.L.). One of the authors (L.W.) is supported by DSTA Singapore under Project Agreement POD0001821. Another author (G.C.) is also partially supported by EU Contract No. HPRN-CT-2000-0156 (QTRANS) and by MURST (Prin 2003, Ordine e caos nei sistemi estesi non lineari: strutture, stocasticita' debole e trasporto anomalo).

${ }^{1}$ J. Bardeen and W. H. Brattain, Phys. Rev. 74, 230 (1948).

${ }^{2}$ M. Terraneo, M. Peyrard, and G. Casati, Phys. Rev. Lett. 88, 094302 (2002).

${ }^{3}$ B. Li, L. Wang, and G. Casati, Phys. Rev. Lett. 93, 184301 (2004).

${ }^{4}$ B. Li, J.-H. Lan, and L. Wang, Phys. Rev. Lett. 95, 104302 (2005)

${ }^{5}$ V. L. Pokrovsky and A. L. Talapov, Theory of Incommensurate Crystals, Soviet Scientific Reviews Supplement Series, Physics Vol. 1 (Harwood, New York, 1984).

${ }^{6}$ O. M. Braun and Y. S. Kivshar, Phys. Rep. 306, 1 (1998).

${ }^{7}$ D. G. Cahill, W. K. Ford, K. E. Goodson, G. D. Mahan, A. Majumdar, H. J. Maris, R. Merlin, and S. R. Phillpot, J. Appl. Phys. 93, 793 (2003).

${ }^{8}$ R. Besseling, R. Niggebrugge, and P. H. Kes, Phys. Rev. Lett. 82, 3144 (1999).

${ }^{9}$ S. C. Erwin, A. A. Baski, L. J. Whitman, and R. E. Rudd, Phys. Rev. Lett. 83, 1818 (1999). 